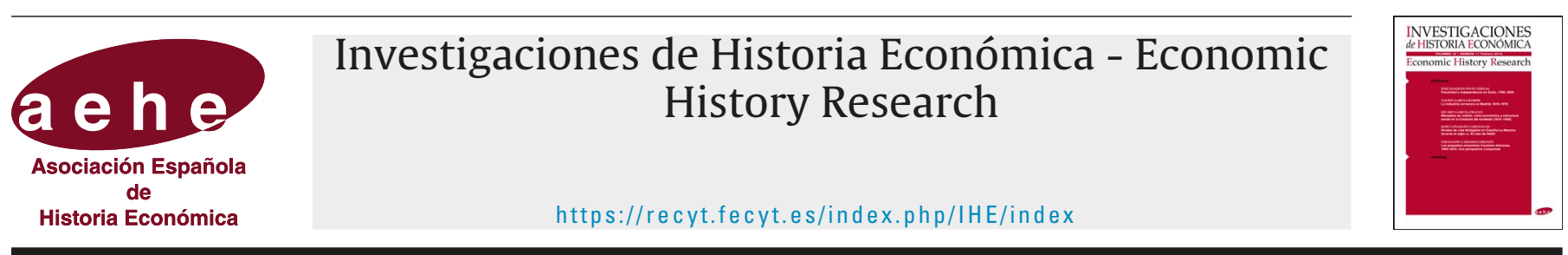

Artículo

\title{
La depreciación argentina durante la Gran Depresión. ¿Por qué falló la teoría?
}

\section{Javier Encabo González*}

Colegio de México. Estudiante de doctorado

\section{INFORMACIÓN DEL ARTÍCULO}

Historia del artículo:

Recibido el 1 de junio de 2017

Aceptado el 12 de septiembre de 2018

On-line el 4 de febrero de 2020

\section{Códigos JEL:}

E31

E42

F33

N16

Palabras clave:

Economía argentina

Patrón oro

Gran Depresión

Pass-through

\begin{abstract}
R E S U M E N
El objetivo de este artículo es analizar las condiciones en las que se produjeron las depreciaciones en Argentina durante la Gran Depresión. El método: una revisión de la literatura y la aplicación de análisis econométrico. Para ello, tras analizar el contexto monetario de la época y el impacto de la Gran Depresión, se abordan las condiciones económicas en América Latina y Argentina durante aquellos años. Las literaturas internacional y nacional destacan que, frente a las dificultades de efectuar devaluaciones internas para ajustar los precios, la depreciación monetaria fue crucial para el reequilibrio de precios. Esto se logró al contrario de lo que la teoría de la paridad de poder adquisitivo hubiera predicho. A través de técnicas econométricas, se verá cómo los precios de las exportaciones argentinas fueron claves para el éxito de esta medida.
\end{abstract}

C 2018 Asociación Española de Historia Económica.

\section{Argentinian Depreciation during the Great Depression. Why did theory fail?}

\author{
A B S T R A C T
}

The aim of this paper is to analyse the conditions in which depreciations in Argentina during the Great Depression were produced. The method: an analysis of the literature and the development of an econometric analysis. To this effect, after analysing the monetary context in those years and the impact of the Great Depression, the economic conditions in Latin America and Argentina are presented. National and International literatures highlight that, against the difficulties to proceed with internal devaluations to adjust prices, monetary depreciation was crucial to rebalance prices. This was achieved on the contrary of what would be expected according to the Purchasing Parity Theory. Through econometric techniques, the prices of Argentinean exports will be shown as a key for the success of this measure.

\footnotetext{
- Autor para correspondencia.

Correo electrónico: javier.encabo@hotmail.com (Javier Encabo González)
} 


\section{Introducción}

Varios años han pasado desde el comienzo de la Gran Recesión en el año 2008. Consecuentemente, el mundo académico ha buscado entender con mayor profundidad los orígenes de la misma. El recurso a la historia ha sido uno de los métodos empleados. En este punto, es inevitable vincular la Crisis del 2008 con la Gran Depresión de los años de 1930. Artículos en revistas y periódicos (Eichengreen and O'Rourke, 2010; The Economist, 2011), artículos académicos (Temin, 2010), y libros (Eichengreen, 2015) han defendido esto. Para los mencionados autores, varias son las características compartidas de ambos episodios. En primer lugar, la magnitud de la caída de la producción y su extensión a nivel global fue excepcional. En segundo lugar, las dos fueron crisis de tendencia deflacionista y las economías occidentales fueron las más expuestas. Finalmente, ambas se expandieron a través de los circuitos financieros y afectaron gravemente a la economía real.

Cuando llegó esta Gran Recesión, Europa estaba a punto de culminar una década del experimento económico más importante hasta la fecha: la adopción del euro como moneda única. Con ella, los países miembros habían cedido su soberanía monetaria en este proyecto de tipo de cambio fijo. Poco a poco, se fueron formando desequilibrios de precios entre las economías europeas. Durante la crisis del 2008, las periféricas fueron las que más sufrieron dichos desequilibrios provocando la crisis del euro de 2010. Desprovistas de política monetaria, el camino obligado fue acometer devaluaciones internas para reequilibrar los niveles de precios con respecto a los países del norte. ¿Pudo haberse hecho esto de alguna otra forma?

Ante esta cuestión problemática, América Latina durante la Gran Depresión se muestra como una experiencia pasada desde la que poder aproximarse a una respuesta. Al comienzo de la misma el sistema monetario mundial estaba dominado por el patrón oro, un tipo de cambio fijo. También se estaba produciendo una crisis deflacionista a nivel mundial. América Latina formaba parte de la periferia de dicho sistema económico y, ante las dificultades, la región sí optó por desengancharse del sistema monetario. Este artículo aborda el caso de Argentina, donde el patrón oro se abandonó muy rápidamente y se permitió una veloz depreciación de la moneda. Este fue un proceso relativamente exitoso, ya que el país, así como la región en general, lograron una recuperación más rápida que la del resto de países occidentales. ¿Cuáles fueron las causas de dicho éxito? Profundizar sobre esta cuestión puede contribuir, aunque sea en cierta medida, a arrojar luz sobre la problemática reciente.

Tras subrayar el contexto monetario internacional y el impacto de la Gran Depresión en Argentina, se procederá a estudiar la depreciación de la moneda en la economía argentina. Esto contribuyó al reequilibrio de los precios relativos del país a nivel internacional. Se efectuará un primer análisis empírico para cuantificar el pass-through (impacto de la depreciación de la moneda sobre el nivel de precios) en la economía argentina durante la Gran Depresión. La literatura ya ha hablado de este positivo reequilibrio, pero no ha entrado a profundizar en las raíces de este. Siguiendo la teoría de la paridad de poder adquisitivo (PPA), estas depreciaciones podrían haber llevado a episodios inflacionarios. En el país esto no ocurrió. Por ello, se procederá a efectuar un segundo análisis en el que se trata de dilucidar por qué esto fue así.

El artículo se estructura de la siguiente forma: tras esta introducción, la siguiente sección se centra en analizar el con- texto monetario internacional de la época y su relación con la Gran Depresión. Después, hay un pequeño apartado sobre el impacto de esta en América Latina. En cuarto lugar, se dedica una sección al caso concreto de Argentina y el contexto en el que se dio. A continuación, se desarrollan dos análisis empíricos. El primero, para el cálculo del pass-through. El segundo, para analizar las raíces de la deflación argentina que permitieron un cómodo abandono del patrón oro. Finalmente se añade un apartado a modo de conclusión.

\section{El contexto monetario internacional}

La Primera Guerra Mundial supuso un lapso en el sistema monetario internacional que había imperado desde los años de 1870, el patrón oro. Los años del conflicto y los inmediatamente posteriores fueron de gran inestabilidad en lo económico. Esto provocó un deseo generalizado de vuelta al patrón oro, símbolo de la prosperidad económica previa (Kemmerer, 1944, p. 109). Se conformó una visión idealizada de un sistema perfecto, estable y automático (Martín-Aceña and Reis, 2000, p. 1). Tras duros procesos de ajuste, casi todos los países consiguieron retomar lo que se denominó patrón oro de entreguerras. Argentina, así como toda América Latina, no fue una excepción. Tal era el interés, que gran cantidad de países de la región se pusieron en manos de consejeros económicos extranjeros como Edwin Kemmerer u Otto Niemeyer para atraer inversiones y financiación externa (Bordo and Rockoff, 1996). Algunas obras especializadas en este tema son las de Paul Drake (1989) y Emilie Rosenberg (1999). Las recomendaciones resultantes llevaron a la creación de bancos centrales en algunos países, la adopción de políticas de ortodoxia monetaria y fiscal y la vuelta al patrón oro. La característica principal del mismo fue que, dada la escasez de metálico, muchos países volvieron al sistema respaldando sus monedas con reservas de dólares o libras en vez de metal; esto se denominaría el patrón de cambio oro (Bordo, 1993; Bordo y Schwartz, 1999; Eichengreen y Flandreau, 1997; Officer, 2008). El sistema resultante produjo muchas rigideces a nivel internacional y fue incapaz de responder a las tensiones económicas que vendrían a posteriori (Eichengreen, 1992). Estas se desatarían en la Gran Depresión que azotaría al mundo desde finales de la década de 1920.

Los trabajos sobre la relación patrón oro y Gran Depresión son numerosos (Bernanke y James, 1991; Bordo y James, 2010; Della Paolera y Taylor, 1998; Eichengreen, 1992; Eichengreen y Sachs, 1985; Kemmerer, 1944; Prebisch, 1991a; Temin, 1993). Una corriente importante de literatura económica señala la deflación como la principal causa de la Gran Depresión (Bordo y James, 2010; Eichengreen y Temin, 2000; Friedman y Schwartz, 1963; Romer, 1992). Resultó que el patrón oro de entreguerras tenía grandes tendencias deflacionistas (Bernanke y James, 1991, p. 36). Las causas eran que, en primer lugar, había una gran asimetría entre países con déficit comercial y países con superávit. En la práctica, las autoridades monetarias de Estados Unidos y Francia, países con superávit, tenían incentivos para esterilizar sus monedas y acumular oro contagiando la deflación al resto de países. En segundo lugar, se daba un sistema piramidal de reservas según el cual los países periféricos tenían respaldadas sus monedas en libras o dólares. Cuando estos las convirtieron en oro ante el peligro de depreciaciones, la masa monetaria mundial se resintió inmediatamente. En tercer lugar, el poder de la banca central en ese 
momento era insuficiente. Muchos bancos centrales europeos tenían reglas impuestas para evitar inflaciones como las de principios de la década de 1920. El Banco de Francia, debido a sus restricciones estatutarias, esterilizó masivamente las entradas de oro para evitar la inflación interna exportando deflación al exterior.

Según Bernanke y Harold James (1991, p. 44), para entender la profundidad de la Gran Depresión la clave está en localizar los mecanismos de transmisión entre la deflación y la depresión. Los autores mencionan tres: el incremento de los salarios reales, el aumento de los tipos de interés y la quiebra del sistema financiero. Para ellos, los elementos clave a la hora de explicar la deflación fueron las oleadas de quiebras bancarias (Bernanke y James, 1991, pp. 34, 63) y el ahogo de la propia actividad crediticia (ibid., p. 49). Eichengreen y Sachs ponen mayor foco en la influencia de los salarios reales y los tipos de interés (Eichengreen y Sachs, 1985, p. 943). Temin (1993, p. 92) se centra en las crisis financieras como causa de transmisión de la Gran Depresión entre los países. Ante las crisis bancarias de 1931, las autoridades subieron los tipos de interés para mantener la confianza en la convertibilidad de las monedas. Se sucedieron las quiebras bancarias, pero los países no rompieron las reglas del juego y los bancos centrales no actuaron como prestamistas de última instancia. Para Temin, no fue sino hasta el abandono del patrón oro cuando se pudieron tomar las medidas necesarias para superar la crisis. Eichengreen, el autor más crítico con el oro, propugna que la política más deseable hubiera sido una salida del patrón oro y una devaluación a nivel global (Eichengreen y Sachs, 1985, p. 946). Lo último no siempre sucedió, pero como se analiza a continuación, la salida del oro sí fue imprescindible.

\section{América Latina y el impacto de la Gran Depresión}

Para los países latinoamericanos, la Gran Depresión ya había empezado antes del crac del 29. (Palma, 1984, p. 65). Contemporáneamente, Raúl Prebisch describe las repercusiones monetarias que supuso para la región la subida de tipos de interés de la Reserva Federal en la primera mitad de 1928 (Prebisch, $1991 b$, p. 573). Esta acción, que tenía como objetivo rebajar la especulación bursátil, provocó el drenaje de oro en dirección a los Estados Unidos desde todo el mundo. A esto es necesario sumar la evolución cada vez menos favorable de los precios de las materias primas a finales de la década (Thorp, 1998, p. 110). La región se encontraba con unas perspectivas económicas delicadas incluso antes de la crisis de Wall Street en octubre de 1929, pero con la crisis financiera norteamericana la situación se precipitó: cesaron las entradas de capitales a los países de la periferia y los precios de las materias primas colapsaron. El principal reto de los países latinoamericanos fue entonces la drástica disminución del valor de las exportaciones que afectó severamente la actividad económica (Diaz-Alejandro, 1983, p. 6; Thorp, 1998, p. 111).

La situación de América Latina a la hora de afrontar este shock externo se vio muy condicionada por su posición como deudora internacional (Marichal, 1989, p. 12). Concretamente, los años de 1920 fueron una época de gran expansión del endeudamiento latinoamericano, sobre todo con los Estados Unidos El destino de dichos fondos era principalmente la construcción de ferrocarriles y los proyectos de modernización urbana (Marichal, 1989, p. 217). Esta entrada de capitales extranjeros, sumada a las exportaciones, implicó un flujo de oro de los circuitos inter- nacionales que fue capaz de compensar las salidas en forma de importaciones, intereses y amortizaciones de la deuda externa. A partir del año 1928, esas entradas de oro en forma de deuda ya no compensaban los flujos negativos (Prebisch, 1991b, p. 14). Finalmente, con la congelación de los mercados financieros internacionales un año después, las salidas de oro se dispararon poniendo en grave tensión las balanzas de pagos.

Se ha expuesto lo perjudicial que fue la deflación para el mal comportamiento económico durante la Gran Depresión ¿Cómo fue entonces el comportamiento de los precios en América Latina? Della Paolera y Taylor hablan del caso argentino y de cómo las políticas de redescuento contribuyeron a que no cayera la base monetaria (Della Paolera y Taylor, 1998, p. 4). Para los autores, la destrucción de las expectativas deflacionistas jugó un papel crucial, ya que permitió disminuir los tipos de interés reales fomentando así el consumo y la inversión. Después de un periodo inicial de deflación importada de los Estados Unidos, esta pudo ser revertida (Diaz-Alejandro, 1983, p. 13). Por el contrario, en países con políticas pasivas, como Cuba, el ajuste deflacionario de los precios fue incluso superior al experimentado por los Estados Unidos Prebisch, desde su perspectiva de máxima autoridad económica de su país en ese momento, señalaba cómo la política monetaria más laxa fue la responsable de contrarrestar las presiones deflacionistas provenientes del exterior (Prebisch, 1991a, p. 57).

Las salidas de oro y la drástica caída de las exportaciones pusieron a prueba la capacidad de las economías de la región. Las circunstancias acabaron provocando cambios en las políticas y llevaron a experimentos económicos (Diaz-Alejandro, 1984, p. 22). Siguiendo a Díaz-Alejandro, pueden encontrarse dos tipos de países según las políticas que emplearon: los reactivos y los pasivos (Diaz-Alejandro, 1983, pp. 17-49). Los primeros fueron los países más grandes y con mayor autonomía que se alejaron de la ortodoxia de la época. Entre las medidas que adoptaron se encontraban el abandono de la convertibilidad del oro, la subida de los aranceles, los controles de cambio, las políticas de defensa de los precios de las materias primas exportadas, las medidas de política monetaria (como las devaluaciones o los préstamos de último recurso a la banca) y las moratorias de la deuda. Los países pasivos fueron aquellos que tuvieron mucho menor margen de actuación, siguieron políticas más ortodoxas o tardaron mucho más en tomar medidas como las mencionadas anteriormente. Fue el caso de las pequeñas economías caribeñas y las repúblicas centroamericanas. Sus mayores vínculos económicos y políticos con los Estados Unidos fueron la causa de esta menor autonomía. Que la actuación macro de los países reactivos fue mucho mejor que la de los pasivos goza de amplio consenso (Bértola and Ocampo, 2013; Bulmer-Thomas, 2003; Diaz-Alejandro, 1983; Thorp, 1998). También decir que, aunque esta clasificación es de uso generalizado, si se entra en literatura más concreta sobre cada país durante la crisis, hubo reformas en todas partes, aunque el carácter fuera diferente. Un ejemplo lo tenemos en los países centroamericanos donde si bien el proteccionismo industrializador no se extendería tanto, la Gran Depresión fue una oportunidad aprovechada para la política sustitución de importaciones agrícolas de consumo (Drinot et al., 2015, p. 241). Por lo tanto, el término reactivo no debe llevar a la falsa creencia de que ciertos países fueron dinámicos y otros carentes de reformas. Más bien, el término debe emplearse para hablar del diferente ritmo y profundidad en las que dichas reformas fueron llevadas a cabo. 


\section{La situación en Argentina durante la Gran Depresión}

Argentina puede ser considerada parte de estos países reactivos. Las reformas llevadas a cabo no pueden entenderse sin el gran impacto de la Gran Depresión en la economía austral. El desplome de la financiación externa y la caída del valor de las exportaciones, tanto por caídas en los precios como por cuantía de producto (Ferreres, 2005, pp. 611-623), afectaron gravemente a la economía argentina. Esto se tradujo en un alza del desempleo, la restricción de las importaciones y, por lo tanto, en un deterioro del nivel de vida de la población. Los grupos más afectados fueron sobre todo los más vulnerables, desde los trabajadores rurales a los obreros urbanos. Pero las clases medias urbanas también se vieron perjudicadas. Este hecho provocó una pérdida de apoyo político al gobierno de la Unión Cívica Radical, presidido por Yrigoyen, por parte de su base fundamental de electores. Por lo tanto, el contexto económico alimentó una inestabilidad política que se tradujo en el golpe de Estado del 6 de septiembre de 1930 que llevó al poder al general Félix Uriburu.

Esta "revolución conservadora» tenía como objetivo la implementación de políticas diferentes a las llevadas a cabo hasta el momento. La política fiscal fue parte de este cambio. Las acciones hasta ese año había ido en la dirección de refinanciar la deuda flotante con deuda externa a corto plazo a la espera de una mejora de la salud de los mercados financieros (Peters, 1934, p. 158). La nueva administración se puso como objetivo prioritario encauzar un déficit público que había llegado a máximos en dicho año (Della Paolera y Taylor, 1998, p. 12). Autores como Hueyo (1938, p. 288), fueron testigos y protagonistas de dichos cambios. Para ello, la política fiscal que se implementó fue restrictiva. Las medidas adoptadas fueron tanto de incremento de los ingresos como de reducción de los gastos. Se crearon nuevos tributos, se subieron las tarifas arancelarias y se redujeron los salarios de los empleados públicos. No hay debate en cuanto a las políticas fiscales restrictivas (Della Paolera and Taylor, 1998; Diaz-Alejandro, 1984; Diaz Fuentes, 1994).

En cuanto a las políticas monetarias, según Díaz Fuentes (1998, p. 458), estas pueden considerarse "complejas, diversas y heterodoxas»; esta última palabra también la emplean Della Paolera y Taylor. Para ellos, Argentina rompió con la ortodoxia durante esos años y marcan como punto de inflexión el mes de abril de 1931 (Della Paolera y Taylor, 1998, p. 20). En ese momento, la Caja de Conversión pasó de un sistema basado en oro a un sistema fiduciario introduciendo redescuentos como forma de aumentar la masa monetaria. Además, en dichas circunstancias de una balanza de pagos muy debilitada, se impulsó un mecanismo de control de cambios. En un primer momento se marcaron prioridades a ciertos grupos de productos como método para limitar las importaciones. Posteriormente, se pasó a limitar las importaciones según el país de procedencia. Según Salera (1941, pp. 61-62), este tipo de medidas durante los primeros años fueron muy improvisadas; las autoridades fueron aprendiendo a base de prueba y error. Sin duda, el paso a la heterodoxia monetaria fue lo que limitó en gran medida el impacto de la Gran Depresión que sí sufrieron otras economías.

El protagonista de este cambio fue sin lugar a dudas Raúl Prebisch (Della Paolera y Taylor, 1998, p. 24). En esta etapa, aun como subsecretario de Hacienda, fue muy activo a la hora de tomar medidas para luchar contra la depresión, especialmente la deflación. Fue un arduo defensor del abandono de la ortodoxia en 1931 a través del mecanismo de redescuentos. Posteriormente también lo sería de la necesidad de crear un banco central (Prebisch, 1991c). El académico contaría además como aliado al ministro de Hacienda Francisco Pinedo. Este fue elegido como sucesor de Alberto Hueyo en agosto de 1933. Su administración puede ser definida como el paso decisivo a la heterodoxia. Entre las medidas que implementó destacaron el impulso a la depreciación de la moneda, la reformulación del control de cambios (con un tipo de cambio libre y otro oficial) y la mencionada apuesta por la creación de un banco central. Tanta fuerza tuvo este personaje, que fue capaz de desechar gran parte de las recomendaciones de Otto Niemeyer (Díaz Fuentes, 1998, p. 453). Este había sido un banquero y funcionario enviado por el Banco de Inglaterra para asesorar la constitución de dicha institución. Para más detalles de la actividad de Niemeyer puede consultarse su informe en la Revista de Economía Argentina (Niemeyer, 1933). Para un análisis más detallado de la administración de Pinedo puede consultarse a Gerchunoff y Machinea (2010, pp. 122-123). Estas fueron las innovaciones más destacadas de la política monetaria durante esos años. Sin embargo, mientras que el trabajo de Della Paolera y Taylor se centra en las políticas de redescuento, el presente artículo va más enfocado a la evolución del tipo de cambio.

Como se ha comentado anteriormente, las tensiones monetarias por la salida de oro afectaron gravemente a Argentina al igual que a toda la región. Aun con unas reservas de oro muy elevadas (pero disminuyendo por la imposibilidad de refinanciar los préstamos al exterior) se tomó la decisión de abandonar el patrón oro a finales de 1929. La experiencia de un tipo de cambio flexible no era algo nuevo para Argentina; había sido, de hecho, algo frecuente en el país. Especialmente tratado ha sido el ajuste que vivió el país austral en un régimen no convertible a finales del xIx. Dicho periodo ya estaba estudiado en los años de 1930 por J. H. Williams (1920) y sería profundizado por Alec Ford (1958). Esto llevó durante un tiempo a la fluctuación de la cotización de la moneda, como puede observarse en la figura 1, que muestra la evolución del valor del peso con respecto a la cotización oficial del peso en oro.

La depreciación que tuvo lugar fue enorme; en año y medio la moneda perdió casi la mitad de su valor. En la gráfica se pueden apreciar tres etapas. En primer lugar, hasta noviembre de 1929, en la que se mantiene una cotización constante del peso con respecto al oro; estabilidad que se había mantenido desde el regreso al patrón oro en 1927. En segundo lugar, desde diciembre de 1929 hasta noviembre de 1931. Durante este tiempo, se produjo una depreciación del peso de hasta un $47 \%$ con respecto a su paridad oficial peso-oro en septiembre de 1931. Finalmente, a partir de diciembre de 1931, la cotización del peso se estabilizó hasta noviembre de 1933. En la figura también están marcados los dos eventos monetarios más relevantes. La línea 1 (L1), en diciembre de 1929, marca el abandono oficial del patrón oro por parte de Argentina (League of Nations, 1935, p. 219). La línea 2 (L2), en abril de 1931, indica el inicio de la política de descuentos de la Caja de Conversión, acción que contribuyó a la devaluación, aunque no en gran magnitud (Della Paolera y Taylor, 1998, p. 33). Como se puede observar gráficamente, dichos eventos fueron puntos de inflexión.

El abandono del patrón oro fue un evento fundamental para la economía argentina. Fue el mismo gobierno populista de Yrigoyen, cuyo partido había abogado por retornar a dicho 


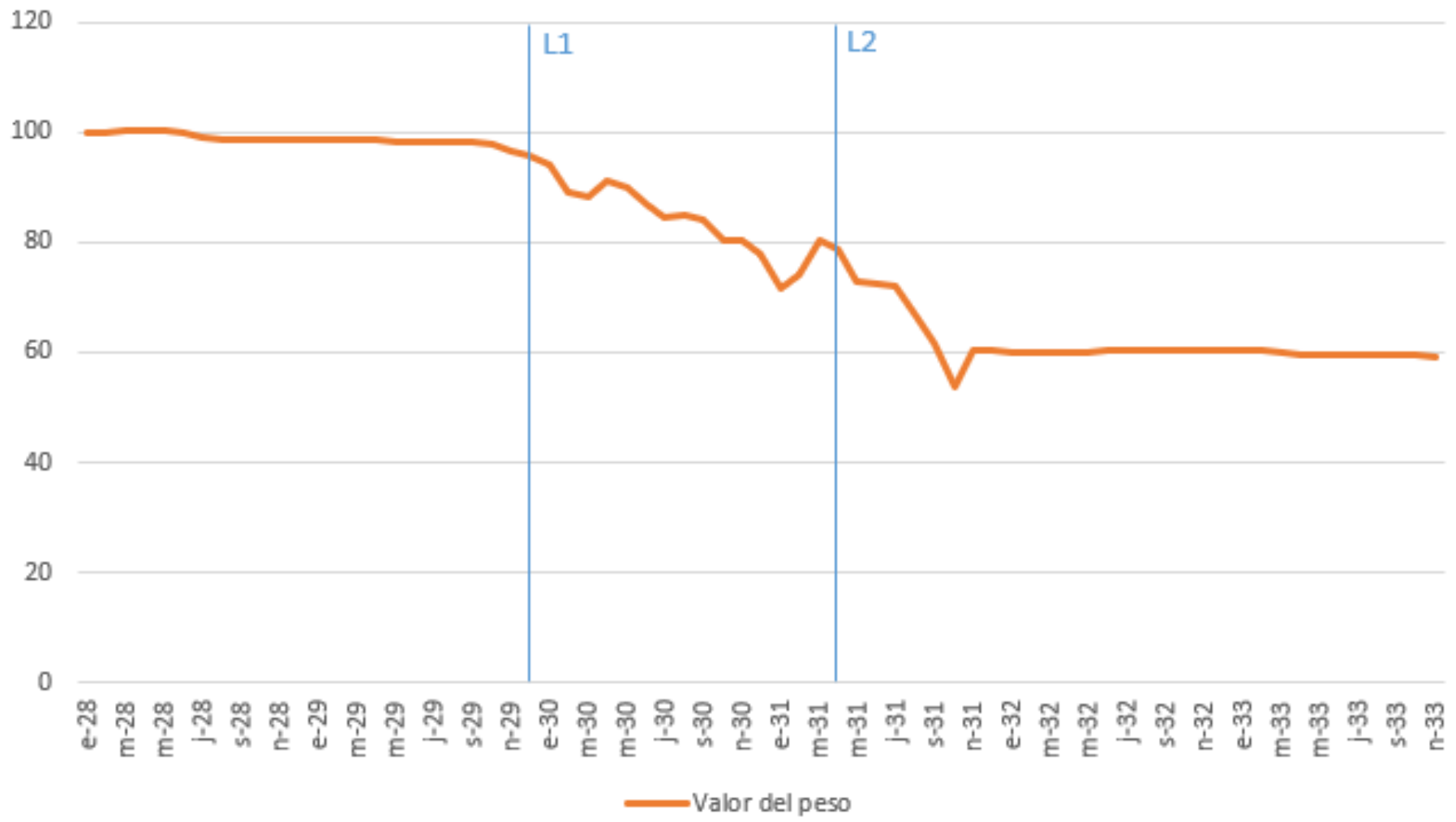

Figura 1. Valor del peso argentino con respecto a su paridad original en el patrón oro, 1928-1933. Base: enero de $1928=100$.

Fuentes: años 1928-29: League of Nations (1931); años 1930-31: League of Nations (1933); años 1932-33: League of Nations (1935).

sistema unos años antes, el que optó por ello. La medida no fue sencilla, pues el régimen seguía siendo considerado como algo incuestionable. De hecho, se abandonó tratando de mantener una administración controlada del tipo de cambio, algo que ya se había llevado a cabo a mediados de la década de 1880 en un anterior abandono del patrón oro (Ford, 1958). A pesar de todo, hubo una devaluación de la moneda, y tuvo efectos positivos. El motivo era que el mecanismo de equilibrio internacional de precios, el mecanismo de ajuste de Hume, permitía en teoría un correcto funcionamiento de la economía internacional. Sin embargo, ello requería tiempo. La depreciación monetaria era, por lo tanto, una posible solución que permitía un rápido ajuste de los niveles de precios (Eichengreen y Portes, 1989). Además, los niveles de precios de productos transables (agropecuarios) eran muy volátiles en Argentina. Siguiendo esta lógica, debido al gran derrumbe de los precios de las exportaciones desde 1928, los ajustes de los precios internos habían de ser también muy severos. Al tiempo que esto acontecía, Prebisch era perfectamente consciente de las dificultades de efectuar dicha deflación interna dadas las rigideces salariales y contractuales normales en la economía argentina (Prebisch, 1991a, p. 59). Además, el peligro de esta medida era caer en una espiral nociva para la economía en la que la que las continuas caídas de precios y las expectativas deflacionistas incrementaran los tipos de interés reales, desalentaran el consumo y la inversión y, por lo tanto, profundizaran la depresión. Esto era lo que estaba ocurriendo en unos Estados Unidos que, al mismo tiempo, exportaba su deflación a todo el mundo.

En cualquier caso, la devaluación del peso benefició a la economía argentina por doble vía: por un lado, contribuyó a mitigar la deflación importada del exterior y sus perniciosas consecuencias; por otro, permitió ajustar los precios con respecto al exterior compensando, entre otras cosas, la caída de los precios internacionales de sus productos de exportación. Esto es lo que nos dice la teoría económica, lo señala la literatura actual e incluso el resultado fue apreciado por los académicos contemporáneos: Peters (1934, pp. 162-163), desde los Estados Unidos, alababa los beneficios que había acarreado para Argentina. Encontrar las raíces de este fenómeno es el objetivo de la parte empírica que viene a continuación.

\section{Un análisis empírico: el pass-through y la evolución de los precios de Argentina entre 1930 y 1933}

Como ya se ha señalado, según Prebisch la depreciación monetaria fue el factor clave que permitió el ajuste de los precios. En esta parte empírica del trabajo se trata de establecer una relación entre las depreciaciones de la moneda ocurridas en Argentina, fruto de la salida del patrón oro, con la evolución de los niveles de precios. Como se podrá observar, los precios no siguieron la lógica económica que cabría esperar. Por lo tanto, una segunda parte tratará de averiguar por qué fue así.

5.1. Concepto de la paridad de poder adquisitivo y su incumplimiento en América Latina

En primer lugar, es necesario partir de la teoría económica y fijarse en PPA. Según Taylor y Taylor: «La paridad del poder 
120

100

80

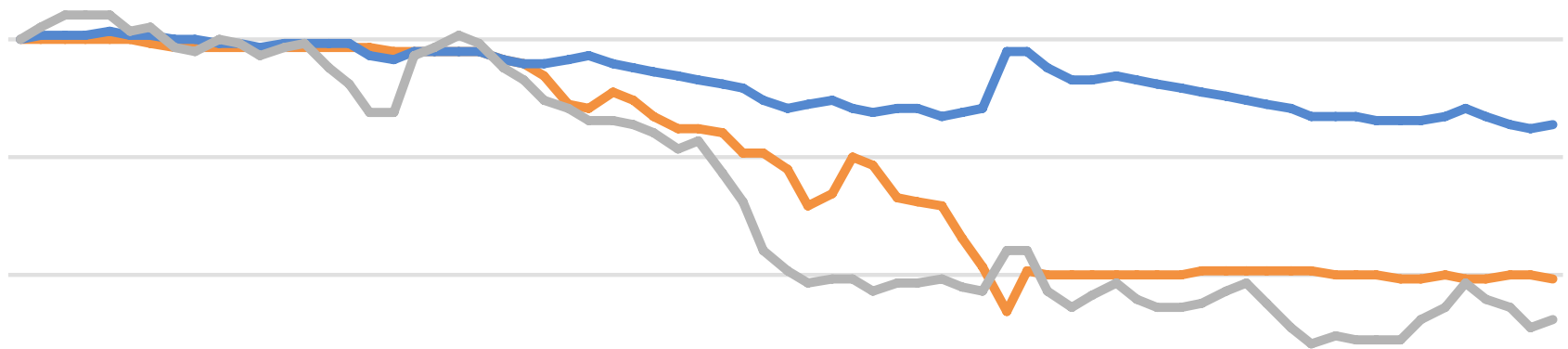

40

20

0

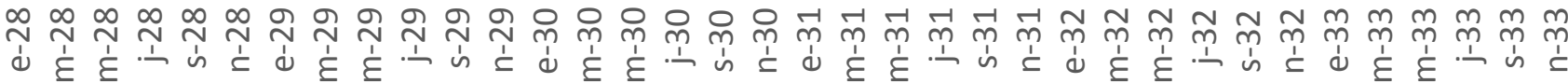

Valor del peso —índice de precios generales

Índice de precios agropecuarios

Figura 2. Índice general de precios, de precios agropecuarios y el valor del peso en oro de Argentina, 1928-1933. Base: Enero 1928 = 100.

Fuentes: Precios: Prebisch (1991d) Tipo de cambio: años 1928-29: League of Nations (1931); años 1930-31: League of Nations (1933); años 1932-33: League of Nations (1935).

adquisitivo es una teoría que dicta que el tipo de cambio nominal entre dos monedas debería ser igual a la ratio del nivel agregado de precios entre los dos países, de tal forma que una unidad de cuenta en un país debería tener el mismo poder adquisitivo en el extranjero" (Taylor y Taylor, 2004, p. 1). Partiendo de dicho razonamiento, las variaciones de los tipos de cambio entre dos países no podrán influir en los precios relativos de los bienes transables y, por lo tanto, para dichos bienes las depreciaciones monetarias no pueden ser utilizadas para ganar competitividad. Además, las depreciaciones monetarias llevarán al encarecimiento de los productos extranjeros y esto supondrá inflación generalizada. Esta es una teoría ampliamente estudiada y paradójica: hay consenso en que la PPA se cumple a largo plazo, pero no hay certeza de que a corto plazo sea así.

En el caso de América Latina en el periodo de la Gran Depresión, hay evidencias de que las depreciaciones monetarias no se tradujeron en episodios inflacionarios (véase tabla 1 ). Solo Chile entre 1929 y 1930 vio como los precios subieron al tiempo que se producía una depreciación del peso. Esto es de interés, pues siguiendo la teoría la región habría conseguido ventajas sustanciales en el tipo de cambio real sin haber incurrido en los costes de inflación. Cabe tener en cuenta la importancia de las presiones deflacionistas del contexto global y la caída generalizada de la oferta monetaria en la región -véase en Díaz-Alejandro (1983, p. 17)-. En cualquier caso, América Latina consiguió a través de las devaluaciones monetarias lo que no consiguió en el sistema del patrón oro: ajustar sus precios relativos con respecto a la economía internacional.

\section{Tabla 1}

Las depreciaciones en América Latina y el comportamiento de los precios

\begin{tabular}{rrr|rr|rr|rr|rr} 
& \multicolumn{2}{c|}{ Argentina } & \multicolumn{2}{|c|}{ Brasil } & \multicolumn{2}{|c|}{ Chile } & \multicolumn{2}{|c|}{ Colombia } & \multicolumn{2}{|c}{ Perú } \\
\cline { 2 - 11 } & $\mathbf{1}$ & $\mathbf{2}$ & $\mathbf{1}$ & $\mathbf{2}$ & $\mathbf{1}$ & $\mathbf{2}$ & $\mathbf{1}$ & $\mathbf{2}$ & $\mathbf{1}$ & $\mathbf{2}$ \\
\hline 1929 & 100 & 100 & 100 & 100 & 100 & 100 & 100 & 100 & $100^{*}$ & 100 \\
\hline 1933 & 60 & 89 & 54 & 78 & 49 & 179 & 70 & 55 & 41 & 97 \\
\hline
\end{tabular}

* Año base 1930

1 = Índice del tipo de cambio respecto al dólar. Fuente: League of Nations, 1934. 2 = Índice general de Precios. Fuente: Thorp, 1984, p. 336.

Para medir esto el indicador más conocido es el pass-throu$\mathrm{gh}$. El pass-through es un cálculo que mide el efecto de las variaciones del tipo de cambio sobre el nivel de los precios. Cuando el indicador es superior a uno, cada punto en la devaluación provoca una cifra mayor de inflación. Cuando el indicador es uno, cada punto de devaluación se transmite íntegro a la inflación. Cuando el pass-through es próximo a cero, cada punto en la devaluación de una moneda se traduce en poca inflación.

Antes de aplicar el modelo, puede observarse en la figura 2 la evolución del valor del peso y los precios durante la Gran Depresión en Argentina. En el mismo se observa un comportamiento dispar del valor de la moneda y los precios, en contradicción con la PPA y en consonancia con lo dicho anteriormente. No es hasta octubre de 1931 cuando parece incrementarse la 
inflación acorde a las depreciaciones de la segunda mitad del año, pero ese repunte ni siquiera es persistente.

Volviendo a la teoría para explicar el no cumplimiento de la PPA, tenemos que decir que en Engel (2002, pp. 4-6), se analizan multitud de explicaciones de líneas teóricas que tratan de dar respuesta a este problema. Algunas son, por ejemplo, el local currency pricing. Según esta teoría, si una empresa comercializa sus productos internacionalmente, es posible que, aunque se produzcan variaciones en el tipo de cambio, los precios en el país de destino no vayan a cambiar. La razón es que los precios fijados para el mercado exportador pueden formar parte de estrategias de segmentación o posicionamiento en el mercado que hacen más rentable asumir las pérdidas del tipo de cambio antes que cambiar los precios. También es importante tener en cuenta que hay otros costos que forman parte del producto importado y no pueden ser explicados por el tipo de cambio: costos de transporte, marketing, distribución o comercialización. Por otra parte, se puede dar el caso de que los bienes importados sean productos intermedios de un proceso de producción mayor, como en los componentes de la industria automotriz. En este caso, el impacto del tipo de cambio sobre los precios dependerá de la capacidad o no de los productores nacionales de componentes de proveer bienes sustitutivos de los importados. Estos son enfoques muy adecuados para economías con un tipo de comercio más actual y sofisticado. Tal vez no lo sean tanto para explicar cómo funcionaba el comercio internacional de Argentina en los años de 1930; en este, el local currency pricing se hace muy complicado y no había capacidad de sustituir a corto plazo muchos bienes intermedios. Sin embargo, entre las explicaciones de Engel se asoma algo que puede dar una pista de lo que pasó en aquellos años. Es el asunto de los bienes de importación. En aquellos años, el país austral importaba gran cantidad de bienes de consumo y bienes intermedios de producción que cada vez resultaban más baratos debido a la deflación generalizada (Ferreres, 2005, p. 625). Engel, señalando que el no cumplimiento de la PPA puede explicarse por el lado de las importaciones, da una pista de cual pudo ser la causa del anómalo comportamiento de los precios en la Argentina de esos años. En la última parte del artículo se retomará este punto.

\subsection{Fuentes}

Para elaborar el modelo se han empleado tres índices de precios: uno con los precios argentinos, otro con los estadounidenses y otro con los británicos. La frecuencia es mensual y va desde enero de 1930 a noviembre de 1933 . Además, se ha construido otro con el valor del peso argentino con respecto al oro con la misma frecuencia y que comprende las mismas fechas. Para este índice se han utilizado datos de los anuarios estadísticos de la Sociedad de Naciones desde 1928 a 1936. Son cifras elaboradas contemporáneamente a los acontecimientos y muy similares a las empleadas en publicaciones de actualidad como Gómez (2018, p. 142). Los índices de precios de Argentina empleados en este estudio (precios generales, precios agropecuarios y no agropecuarios) provienen de otra fuente, la Revista Económica. Revista Económica, que fue una publicación de la Oficina de Investigaciones Económicas del Banco de la Nación Argentina surgida en 1928. Es importante tener en cuenta que dicha publicación fue muy importante como antecedente de posteriores trabajos del Banco Central de Ar- gentina. Dada la labor que Raúl Prebisch realizó ahí, su confiabilidad es aceptada. Se ha accedido a ellos a través de un tomo recopilatorio con las obras de Raúl Prebisch (1991d, p. 228). Para los precios del Reino Unido, se han empleado también los anuarios estadísticos de la Sociedad de Naciones. Para el caso de los Estados Unidos, los precios proceden del libro Consumer's Prices de Robert A. Sayre (1948, pp. 37-38). A partir de los índices de precios y tipos de cambio se han elaborado las variaciones mensuales, la cifra empleada en este modelo (datos disponibles en el Apéndice Estadístico). Para el tipo de cambio se ha tomado la variación del valor del peso argentino. Esto quiere decir que cuando el valor aumenta la moneda se está apreciando, y cuando disminuye se está depreciando. Este detalle es importante a la hora de efectuar una interpretación de los resultados.

\subsection{Primer modelo: el análisis del pass-through en Argentina ¿Se cumplió la teoría de la paridad poder adquisitivo?}

Para cuantificar el pass-through se ha usado un modelo empleado por Dani Rodrik y Kathryn Domínguez aplicado a Bolivia en el estudio de la hiperinflación de los años de 1980 (Dominguez y Rodrik, 1990). Un buen resumen del mismo y una comparativa entre otros similares para el caso boliviano encontrase en Cupé (2002, pp. 3-8). La idea que hay detrás del mismo es la comprensión de la depreciación de la moneda como un componente crucial de la inflación en una economía abierta. Según la regresión propuesta, la inflación de un mes es el resultado de la inflación del mes anterior, la depreciación de la moneda de dicho mes y la depreciación del mes previo. Todo ello son variables expresadas en términos de variación mensual. El objetivo del empleo de este modelo es conocer el impacto de las depreciaciones de la moneda sobre los precios, es decir, el pass-through. El modelo es el siguiente:

$\mathrm{n}_{\mathrm{t}}=$ Constante $+\alpha \cdot$ Inflación $_{\mathrm{t}}-1+\beta \cdot$ Depreciación $_{\mathrm{t}}+\delta \cdot$ Depreciación $_{\mathrm{t}}-1$

El pass-through serían las beta y delta resultantes. Las características que destacan de dicho modelo son la simplicidad y el empleo de cifras mensuales. La simplicidad tiene como punto a favor ser realmente un modelo asequible para los datos disponibles de la década de 1930. Como punto negativo, se observa una excesiva sencillez en un contexto de modelos alternativos en los que influyen más variables como la tasa de apertura comercial, la emisión de moneda o la inflación internacional (Cupé, 2002, p. 7). El empleo de cifras mensuales permite analizar episodios puntuales de forma más concreta que si fueran anuales. Además, esto nos permite multiplicar por doce la muestra de las regresiones, algo que ayuda a que el modelo funcione mejor. Así, el resultado del análisis econométrico ha de ser interpretado con cautela. Sin embargo, el resultado puede servir para analizar hasta qué punto el pass-through está alejado de cero o de uno, mostrando así la sensibilidad de los precios a una modificación en el tipo de cambio.

A continuación, se expone el modelo de Domínguez y Rodrik aplicado para Argentina (tabla 2). El modelo ha sido estimado por mínimos cuadrados ordinarios y las series son estacionarias al $1 \%$. Esto último implica que pueden hallarse patrones de comportamiento en las mismas y por lo tanto permite confiar en los resultados del modelo. 
Tabla 2

Resultado del pass-through, 1930-1933

\begin{tabular}{lc}
\hline \multicolumn{2}{l}{ Método OLS. Variable dependiente: Precios generales Argentina } \\
\hline \multicolumn{2}{l}{ Variables independientes } \\
\hline Constante & $-.0057^{*}$ \\
& $(-2.63)$ \\
\hline $\begin{array}{l}\text { Variación de precios del } \\
\text { mes anterior en }\end{array}$ & $0.3576^{*}$ \\
Argentina & $(2.38)$ \\
\hline $\begin{array}{l}\text { Variación del valor del } \\
\text { peso argentino }\end{array}$ & $0.3218^{* *}$ \\
\hline $\begin{array}{l}\text { Variación del valor del } \\
\text { peso argentino en el mes } \\
\text { anterior }\end{array}$ & $(5.08)$ \\
\hline
\end{tabular}

* Significativo al 5\%. ** Significativo al 1\%. Los números entre paréntesis son los estadísticos $t$. Variable dependiente: Inflación de Argentina

Los resultados indican la existencia de una relación estadística significativa entre la variación del valor del peso y la variación de la inflación en Argentina. La cuestión es que el signo es el contrario de lo que predeciría la teoría económica. Esta regresión muestra que por cada punto porcentual que se apreciaba el peso argentino, la inflación aumentaba en un $0,32 \%$. Esta es la cuantía del pass-through sobre los precios en Argentina entre 1930 y 1933. Dicho de otro modo, aun a pesar de la profunda depreciación que vivió la moneda argentina, los pre- cios, en vez de aumentar como predeciría la teoría económica, disminuyeron.

Este resultado del modelo, aunque contradice la PPA, tiene sentido viendo la dirección de los precios y del valor del peso en los años estudiados. Entre las implicaciones de esto, destaca que, por sí sola, la pérdida del $50 \%$ del valor del peso entre enero de 1930 y octubre de 1931 llevó a una ganancia de competitividad mayor que toda la que lograron los Estados Unidos con cuatro años de deflación de precios. Sin embargo, otros episodios en la historia de Argentina y de otros países muestran que las devaluaciones no siempre fueron igual de exitosas. ¿Cuál es la causa de que en este caso sí fuera así? ¿Por qué los niveles de precios siguieron bajando persistentemente? Averiguar las raíces de dicho comportamiento de los precios es el objeto del siguiente modelo.

\subsection{Segundo modelo: el movimiento de los precios en Argentina. ¿El origen de la deflación era externo?}

La inflación y deflación son fenómenos multicausales de gran trascendencia para la política económica. Estos tienen gran impacto en las decisiones de consumo, afectan a la productividad exterior de una economía, influyen en las decisiones de inversión y alteran los tipos de interés reales pudiendo cambiar la rentabilidad esperada de prestamistas o prestatarios. La forma más común de clasificar dichos fenómenos es dividirlos según si su origen está en la economía monetaria o en la economía real. La primera puede afectar a los precios a través de cambios en la base monetaria, en las restricciones en el acceso al crédito o en la propensión al ahorro que lleven a

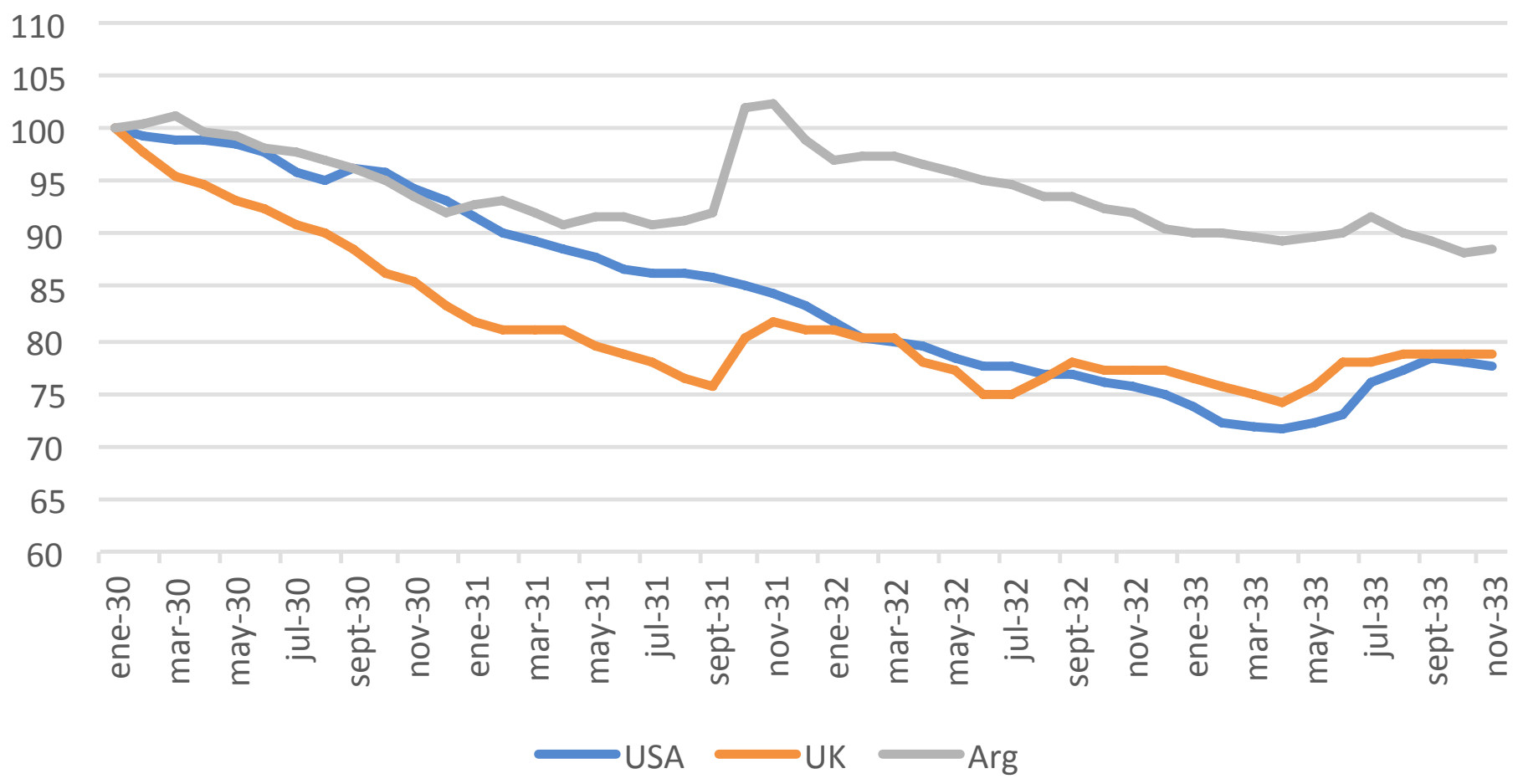

Figura 3. Índice de precios de Argentina, Reino Unido y Estados Unidos, 1928-1933. Base: enero $1928=100$.

Fuentes: precios Argentina: Prebisch (1991d, p. 228). Precios de Reino Unido: League of Nations (1934, p. 249). Precios de los Estados Unidos: Sayre (1948, p.37-38). 
cambios en la masa monetaria no acordes con los cambios en la producción. Por otra parte, cambios de la economía real, como variaciones en los salarios o en la productividad, también pueden implicar cambios en los niveles de precios.

Sin embargo, en este modelo se va a poner el foco en discernir las causas externas e internas de la deflación en Argentina durante la Gran Depresión. Entre las posibles causas internas de la deflación se encuentran las medidas de ajuste llevadas a cabo por la administración de Uriburu, como el aumento de tarifas comerciales o despidos y reducciones de sueldos en la Administración pública. El creciente desempleo y las caídas de salarios en el sector privado también pudieron ser importantes. En una economía con un sector agroexportador tan relevante, la ausencia de malas cosechas pudo tener efectos en los precios. Este último no es el caso, ya que en 1930 las cosechas de trigo fueron muy malas; no hubo, por lo tanto, excedentes extraordinarios de cereal ese año (Gómez, 2018, p. 128; O'Connell, 1984, p. 490). Entre las causas externas destaca principalmente la espiral deflacionista que se desató en todo el mundo en esos años. A continuación, hay una gráfica (figura 3) en la que se puede apreciar la evolución de los precios al por mayor en Argentina, Estados Unidos y el Reino Unido. Puede observarse la gran caída de los precios que sufrieron los dos últimos, más profunda que lo vivido en Argentina. La tesis ampliamente refrendada por el mundo académico es que esta gran deflación se originó en los Estados Unidos y se fue expandiendo a todo el mundo a través del sistema monetario.

Sin embargo, a continuación, se pretende poner a prueba empíricamente si realmente influyó la deflación externa en los precios de Argentina. Algunos trabajos que se han tomado como referencia para este análisis son los de Ha et al. (2003), N'Diaye (2003) y Ocampo (2000, p. 222). El modelo econométrico de Ocampo es en el que se basa metodológicamente el modelo empleado y consiste en el cálculo de los determinantes de la inflación en Colombia entre 1881 y 1939. El motivo por el que se ha empleado como referencia es que para explicar la inflación el autor recurrió al tipo de cambio y a los precios internacionales. Para el autor, lo que ocurría en los precios de otros países podía ser clave a la hora de explicar los precios en Colombia.

El mismo ejercicio puede hacerse para Argentina. Sin embargo, en vez de emplearse precios internacionales, se ha optado por emplear los precios de sus dos mayores socios comerciales: Reino Unido y los Estados Unidos. El objetivo es poder discernir efectos provenientes de ambas economías. Por lo tanto, a continuación, se presenta una regresión para averiguar las raíces de la deflación argentina entre 1930 y 1933. Según este modelo, se considera que los precios generales de Argentina en aquellos años pueden estar influidos por la variación del peso argentino (otra vez el cálculo del pass-through), por la variación de precios del mes anterior en Argentina, la variación de precios en el Reino Unido y en los Estados Unidos. Una primera regresión comprende el periodo que se extiende desde comienzos de 1930 (abandono del patrón oro) hasta noviembre de 1933. A finales de este último año, bajo la batuta del ministro Pinedo, se consolidó un control de cambios con el que se había acumulado una experiencia notable, se acotó la deflación, se logró un tipo de cambio competitivo y se consiguió poner en práctica un considerable nivel de intervención estatal; además se habían reencauzado las buenas relaciones con el Reino Unido durante ese año. Se ha efectuado también otra regresión que comprende el periodo más específico que va desde el abandono efectivo del patrón oro hasta la implan- tación del control de cambios, cuando llega a su fin la libre flotación en la cotización del peso (desde enero de 1930 hasta noviembre de 1931). El objetivo de este modelo será discernir claramente si la deflación argentina fue importada del exterior o si las raíces fueron internas.

\section{Tabla 3}

Raíces externas de la deflación argentina Método OLS. Variable dependiente: Precios generales

\begin{tabular}{|c|c|c|}
\hline $\begin{array}{c}\text { Variables } \\
\text { independientes }\end{array}$ & Ene 1930 - Nov 1933 & Ene 1930 - Nov 1931 \\
\hline Constante & $\begin{array}{c}-0.0023 \\
(-1.16)\end{array}$ & $\begin{array}{c}0.0032 \\
(0.83)\end{array}$ \\
\hline $\begin{array}{l}\text { Variación de } \\
\text { precios del mes } \\
\text { anterior } \\
\text { Argentina }\end{array}$ & $\begin{array}{c}0.3796^{* *} \\
(3.39)\end{array}$ & $\begin{array}{l}0.2187 \\
(1.68)\end{array}$ \\
\hline $\begin{array}{l}\text { Variación del } \\
\text { valor del peso } \\
\text { argentino }\end{array}$ & $\begin{array}{c}0.3405^{* *} \\
(6.58)\end{array}$ & $\begin{array}{c}0.2903^{* *} \\
(5.58)\end{array}$ \\
\hline $\begin{array}{l}\text { Variación de } \\
\text { precios } \\
\text { Reino Unido }\end{array}$ & $\begin{array}{c}0.5462^{* *} \\
(4.80)\end{array}$ & $\begin{array}{c}0.9193^{* *} \\
(6.55)\end{array}$ \\
\hline $\begin{array}{l}\text { Variación de } \\
\text { precios } \\
\text { Estados Unidos }\end{array}$ & $\begin{array}{c}-0.1267 \\
(-0.74)\end{array}$ & $\begin{array}{c}-0.1552 \\
(-0.47)\end{array}$ \\
\hline
\end{tabular}

* Significativo al 5\%; ** Significativo al $1 \%$. Los números entre paréntesis son estadísticos $t$. Variable dependiente: Precios generales de Argentina (agropecuarios y no agropecuarios)

Una vez elaborado el modelo, entre lo que se puede deducir destaca lo siguiente: en primer lugar, la influencia de la variación de los precios del mes anterior sobre los precios resulta significativa para el periodo completo. Esto significa que para todo el periodo estudiado se producía una persistencia de las perspectivas deflacionarias de los argentinos: más de un tercio de la deflación de un mes se trasladaba al siguiente. Para Della Paolera y Taylor (1998, p. 35) a partir de abril de 1931 estas expectativas comenzaron a destruirse. Sin embargo, puede decirse que en promedio influyeron en todo el periodo. En segundo lugar, este método de cálculo del pass-through arroja las mismas cifras que el utilizado anteriormente, pero destaca que para los años de 1930 a 1931, momento de flexibilidad cambiaria, la sensibilidad del indicador es algo menor. Esto quiere decir que, durante esta época, las depreciaciones del peso fueron acompañadas estadísticamente por una menor caída de precios. En tercer lugar, aunque las cifras arrojadas solo permiten hablar de la influencia de los precios del Reino Unido sobre los precios de Argentina, es importante destacar que para ambos periodos esta resulta significativa y de gran magnitud. Mientras que para el periodo completo el impacto de la deflación en Reino Unido se trasladaba en un 54\% a Argentina, para el periodo de tipo de cambio flexible este impacto era mucho mayor, del $90 \%$. Esto último contradice la teoría económica de que la flexibilidad cambiaria funcionó como amortiguador de las variaciones de precios externas. Finalmente, dicho lo anterior se puede concluir que la deflación externa sí tuvo influencia en los precios de la economía argentina. 
Tabla 4

Transmisión de la deflación externa a través de los precios agropecuarios

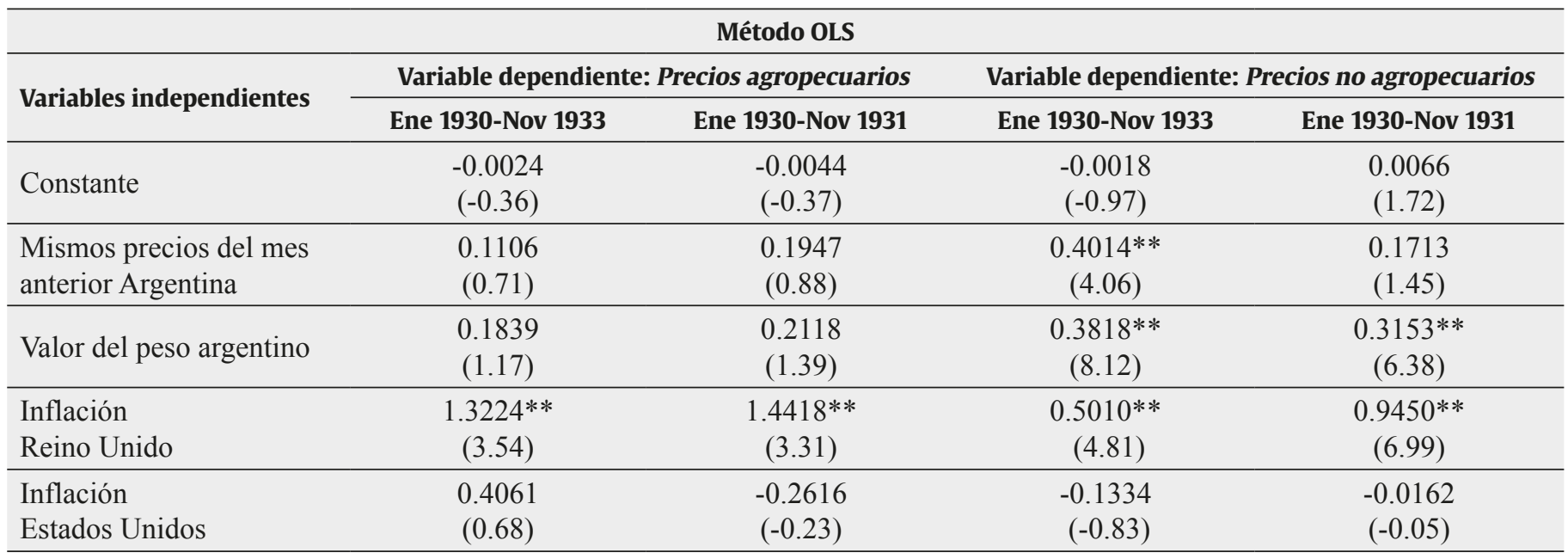

*Significativo al $5 \%$; **ignificativo al 1\%. Los números entre paréntesis son estadísticos $t$.

5.5. Segundo modelo: el movimiento de los precios en Argentina. ¿Fueron los precios agropecuarios transmisores de la deflación?

A continuación, para ir un paso más allá, se va a analizar más en profundidad cuál fue el mecanismo de transmisión de los precios externos. Un primer medio a través del cual pudo trasladarse esta deflación externa fueron las importaciones, algo señalado por Engel (2002). Argentina compraba bienes a otros países que se encontraban en una deflación de precios más profunda. Por ejemplo, los Estados Unidos y el Reino Unido, que suponían el 40\% de las compras en el exterior entre 1929 y 1933 (Ferreres, 2005, pp. 627-635), estaban viendo caer sus precios en un $25 \%$ durante esos años. Sin duda, esto puede explicar que la depreciación del tipo de cambio no llevara a una inflación, pues era compensada por unas importaciones más baratas. Sin embargo, la moneda llegó a depreciarse hasta un 40\% en dichas fechas: la vía de las importaciones es una respuesta insuficiente. Otro posible medio de transmisión de la deflación son las exportaciones de Argentina al exterior. Para estudiarlo se propone a continuación un análisis centrado en los precios agropecuarios en Argentina, el gran producto de exportación.

La tabla 4 es la aplicación del mismo modelo anterior, pero con la variación de los precios agropecuarios y no agropecuarios como variables dependientes. El objetivo es poder examinar el diferente comportamiento de los dos tipos de precios. Se puede observar cómo las presiones deflacionarias del Reino Unido se trasladaban con gran fuerza a los precios agropecuarios en ambos periodos estudiados. Comparado con el resto de los precios de la economía, la deflación agropecuaria fue mucho mayor y el origen de esta era mucho más foráneo (los precios del Reino Unido se trasladaron casi con tres veces más fuerza sobre los precios agrarios y ganaderos que sobre el resto de los precios para todo el periodo estudiado). Es lógico si se conciben los bienes agropecuarios como los más transables y flexibles en una economía agroexportadora como la argentina. Por lo tanto, hay argumentos para pensar que, efectivamente, los precios agropecuarios fueron transmisores de la deflación importada. Destaca también que esa transmisión de la deflación se cumplió incluso en el periodo en el que los tipos de cambio fueron flexibles.

Se saca una conclusión clave de esto último. Si los tipos de cambio flexibles no tuvieron un efecto amortiguador de las presiones deflacionarias externas, es necesario encontrar cuál fue ese mecanismo intermedio que hizo que la deflación británica impactara en los precios de Argentina. La respuesta la tenemos en los precios agropecuarios. Los precios de estos cayeron empujados por la deflación del Reino Unido, el mayor cliente, y por la deflación generalizada de ese tipo de productos en todo el mundo. Esta caída fue incluso superior a la depreciación del peso argentino (véase tabla 5). Por lo tanto, aunque teóricamente el tipo de cambio depreciado permitía aumentar la cantidad de pesos recibidos en Argentina por libras de exportaciones agropecuarias, este efecto se compensaba con creces por la caída de la cantidad de libras recibidas por unidad de producto exportado. Por tanto, los precios de los productos argentinos no solo disminuían en libras, sino también en pesos, aunque en menor medida. Esta caída se acabó trasladando al resto de productos de la economía, muy conectada al sector agroexportador. Así, aunque las importaciones cada vez más baratas hacia Argentina fueron relevantes, las propias exportaciones del país austral también transmitieron la deflación desde el exterior.

\section{Tabla 5}

Comparativa de precios del trigo, los precios agropecuarios de Argentina y la depreciación del peso. Años 1930-1933

\begin{tabular}{cccc}
\hline Año & $\begin{array}{c}\text { Valor } \\
\text { del peso }\end{array}$ & $\begin{array}{c}\text { Precios } \\
\text { internacionales } \\
\text { del trigo }\end{array}$ & $\begin{array}{c}\text { Precios } \\
\text { agropecuarios } \\
\text { Argentina }\end{array}$ \\
\hline 1930 & 100 & 100 & 100 \\
1931 & 79.46709 & 60 & 74.27 \\
1932 & 69.57838 & 50 & 68.8 \\
1933 & 68.16092 & 58.33333 & 66.24 \\
\hline
\end{tabular}

Fuente: valor del peso: League of Nations (1934). Precios trigo: Moxlad, $<$ http://bit.ly/3anIbnU>. Precios agropecuarios Argentina: Prebisch (1991d, p. 228). 


\section{Conclusiones}

La Gran Depresión fue una época de gran excepcionalidad económica. Entre sus implicaciones más espectaculares se encuentra la gran deflación que sufrió la economía mundial en esos años. Por sí sola, esta ha sido empleada para justificar que no se cumplieran teorías como la de la PPA. Gracias a ello la economía argentina pudo aprovechar para depreciar su moneda y contribuir así a su recuperación económica. En este punto, tanto la literatura nacional como internacional sobre la Gran Depresión están de acuerdo: la salida del patrón oro y la depreciación eran las opciones más adecuadas. Sin embargo, este estudio ha tratado de ir más allá. Profundizando un poco más, es necesario ser consciente de que la gran ventaja que sacó Argentina fue la caída tan fuerte de los precios agropecuarios en los mercados internacionales y la gran exposición de su economía a este sector exportador. La transmisión de la deflación a través de la exportación de bienes agropecuarios fue un mecanismo intermedio clave que realmente hizo que no se cumpliera la teoría de la PPA.

Volviendo a la problemática inicial sobre la crisis del euro y las alternativas en política económica a las devaluaciones internas, el estudio del caso argentino revela que no bastó simplemente con un contexto deflacionario mundial de gran magnitud. También fue el contexto de gran apertura comercial y de gran exposición de Argentina a dichas tendencias deflacionarias internacionales lo que permitieron emplear devaluaciones externas con éxito. Esto puede resultar realmente paradójico, una especie de "paradoja de la apertura». Esto se debe a que podría parecer que un país muy abierto y sensible a la deflación global se vería más perjudicado que el resto ante los efectos perniciosos de la misma. Sin embargo, se dio todo lo contrario: el ser muy abierto le permitió a Argentina acometer devaluaciones de la moneda mayores que otros países sin temor a perder competitividad frente a ellos a causa de una teórica inflación que nunca llegó.

\section{Agradecimientos}

Este documento es resultado de mi proyecto final del master interuniversitario de Historia Económica por la UB, UAB y Unizar. Un agradecimiento a todos los docentes, colaboradores y compañeros del máster por todo el buen trato recibido, la ayuda y por las oportunidades que su trabajo ha abierto para mi futuro. Quiero agradecer especialmente a Yolanda Blasco todo el tiempo y esfuerzo que ha dedicado como tutora de este proyecto.

\section{Bibliografía}

Bernanke, B. S. y James, H., 1991. The gold standard, deflation, and financial crisis in the Great Depression: An international comparison. En: Hubbard, G. (ed.). Financial Markets and Financial Crises. University of Chicago Press, Chicago, pp. 33-68.

Bértola, L. y Ocampo, J. A., 2013. El desarrollo económico de América Latina desde la independencia. Fondo de Cultura Económica, México, D. F.

Bordo, M. D., 1993. The Gold Standard, Bretton Woods and Other Monetary Regimes: A Historical Appraisal. NBER Working Paper Series, Cambridge.

Bordo, M. D. y James, H., 2010. The Great Depression and The Great Recession. En: Past and Present: From the Great Depression of 1929 to the Great Recession of 2009.

Bordo, M. D. y Rockoff, H., 1996. The Gold Standard as a "Good Housekeeping Seal of Approval». The Journal of Economic History. 56, 389-428.
Bordo, M. D., Schwartz, A. J., 1999. Monetary Policy Regimes and Economic Performance: The Historical Record. En: Taylor, J. B. y Woodford, M. (eds.). Handbook of Macroeconomics, Volume 1A. Elsevier, pp. 149-223.

Bulmer-Thomas, V., 2003. The Economic History of Latin America since Independence, 2nd ed. Cambridge University Press, Cambridge.

Cupé, E., 2002. Efecto pass-through de la depreciación sobre inflación y términos de intercambio internos bolivianos. UDAPE, La Paz.

Della Paolera, G., Taylor, A. M., 1998. Economic Recovery from the Argentine Great Depression: Institutions, expectations and the change of macroeconomic regime. NBER Working Paper Series, Cambridge.

Diaz-Alejandro, C., 1984. Latin America in the 1930s. En: Thorp, R. (ed.). Latin America in the 1930s. The Role of the Periphery in the World Crisis. Macmillan Press Ltd., London.

Diaz-Alejandro, C., 1983. Stories of the 1930s for the 1980s. En: Aspe, P., Dornbusch, R. y Obstfeld, M. (eds.). Financial Policies and the World Capital Market. The University of Chicago Press, Cambridge, pp. 5-40.

Diaz Fuentes, D., 1994. Crisis y cambios estructurales en América Latina: Argentina, Brasil y México durante el periodo de entreguerras. Fondo de Cultura Económica, México D. F.

Díaz Fuentes, D., 1998. Latin America during the Interwar Period: The Rise and Fall of the Gold Standard in Argentina, Brazil, and Mexico. En: Latin America and World Economy since 1800.

Dominguez, K. y Rodrik, D., 1990. Manejo del tipo de cambio y crecimiento después de la estabilización: el caso boliviano. UDAPE, La Paz.

Drake, P., 1989. The Money doctor in the Andes: The Kremmeres Missions, 19231933. Duke University Press, Londres.

Drinot, P., Knight, A., Hora, R., Vergara, Á., Contreras, C. y Bucheli, M., 2015. La Gran Depresión en América Latina. Fondo de Cultura Económica, México, D. F.

Eichengreen, B., 2015. Hall of Mirrors. Oxford University Press, Oxford.

Eichengreen, B., 1992. Golden fetters: The Gold Standard and the Great Depression. Oxford University Press, New York.

Eichengreen, B., Flandreau, M. (eds.), 1997. The Gold Standard in Theory and History, 2nd ed. Routledge, London.

Eichengreen, B. y O'Rourke, K. H., 2010. A tale of two depressions: What do the new data tell us? VoxEU. org, 8.

Eichengreen, B. y Portes, R., 1989. Dealing with debt: The 1930s and the 1980s. NBER Working Paper Series, Cambridge.

Eichengreen, B. y Sachs, J., 1985. Exchange Rates and Economic Recovery in the 1930s. The Journal of Economic History. 45 (4), 925-946.

Eichengreen, B. y Temin, P., 2000. The Gold Standard and the Great Depression. Contemporary European History. 9 (2), 183-207.

Engel, C., 2002. The responsiveness of consumer prices to exchange rates and the implications for exchange-rate policy: a survey of a few recent new open-economy macro models. NBER Working Paper Series, Cambridge.

Ferreres, O. J., 2005. Dos siglos de economía argentina (1810-2004): historia argentina en cifras. Fundación Norte y Sur, Buenos Aires.

Ford, A. G., 1958. Flexible Exchange Rates and Argentina, 1885-1900. Oxford Economic Papers. 10 (3), 316-338. Doi:10.1093/res/hgnl58.

Friedman, M. y Schwartz, A. J., 1963. A monetary history of the United States, 1867-1960. Princeton University Press, Cambridge.

Gerchunoff, P., 2010. Circulando en el laberinto: la economía argentina entre la depresión y la guerra (1929-1939). Documentos de Trabajo del IELAT, Alcalá de Henares. Doi:10.1017/CBO9781107415324.004.

Gómez, M., 2018. Avatares de un sistema monetario. La Primera Caja de Conversión argentina y su transformación final en Banco Central (1890-1935). Editorial Teseo, Buenos Aires.

Ha, J., Fan, K. y Shu, C., 2003. The Causes of Inflation and Deflation in Mainland China. Hong Kong Monetary Authority Quarterly Bulletin, Hong Kong.

Hueyo, A., 1938. La Argentina en la Gran Depresión Mundial, 1932-1933. El Ateneo, Buenos Aires.

Kemmerer, E., 1944. Gold and the Gold Standard. McGraw-Hill, New York.

League of Nations, 1935. Statistical Year-Book of The League of Nations. Public Finance, Currency Statistics and Prices. 1934-35. League of Nations, Geneve.

League of Nations, 1934. Statistical Year-Book of The League of Nations. Public Finance, Currency Statistics and Prices. 1933-1934. League of Nations, Geneve.

League of Nations, 1933. Statistical Year-Book of The League of Nations. Public Finance, Currency Statistics and Prices. 1932-1933. League of Nations, Geneve.

League of Nations, 1931. Statistical Year-Book of The League of Nations. Public Finance, Currency Statistics and Prices. 1930-1931. League of Nations, Geneve.

Marichal, C., 1989. A Century of Debt Crises in Latin America: from independence to the Great Depression, 1820-1930. Princeton University Press, Princeton.

Martín-Aceña, P. y Reis, J. (eds.), 2000. Monetary Standars in the Periphery. Paper, Silver and Gold, 1854-1933. Cambridge University Press, Cambridge. 
N'Diaye, P., 2003. Determinants of deflation in Hong Kong SAR (No. 3-250). International Monetary Fund, Washington.

Niemeyer, O., 1933. Informe presentado por el perito británico Sir Otto E. Niemeyer al Ministro de Hacienda de la Nación. Revista de Economía Argentina. $30,187-197$.

O'Connell, A., 1984. La Argentina en la depresión: los problemas de una economía abierta. Desarrollo Económico. 23 (92), 479-514.

Ocampo, J. A., 2000. Variable Monetary Regimes in a Preindustrial Economy: Colombia, 1850-1933. En: Martín Aceña, P. y Reis, J. (eds.). Monetary Standards in the Periphery. Paper, Silver and Gold, 1854-1933. Macmillan Press Ltd, London, pp. 207-252.

Officer, L. H., 2008. Gold Standard. EH.net Encyclopedia.

Palma, J. G., 1984. From an Export-led to an Import-substituting Economy: Chile 1914-39. En: Thorp, R. (ed.). Latin America in the 1930s. The Role of the Periphery in the World Crisis. Macmillan Press Ltd., London, pp. 50-80.

Peters, H. E., 1934. The Foreign Debt of the Argentine Republic. The Johns Hopkins Press, Baltimore.

Prebisch, R., 1991a. La acción de emergencia en el problema monetario, 1932. En: Ferrari, G. y Fernández López, M. (eds.). Raúl Prebisch Obras, 1919-1948. Tomo I. Fundación Raúl Prebisch, Buenos Aires, pp. 42-67.

Prebisch, R., 1991b. El movimiento internacional del oro, 1929. En: Ferrari, G. y Fernández López, M. (eds.). Raúl Prebisch Obras, 1919-1948. Tomo I. Fundación Raúl Prebisch, Buenos Aires, pp. 553-577.

Prebisch, R., 1991c. El Banco Central de la República Argentina. En: Ferrari, G. y Fernández López, M. (eds.). Raúl Prebisch Obras, 1919-1948. Tomo II. Fundación Raúl Prebisch, Buenos Aires, pp. 354-395.
Prebisch, R., 1991d. El momento presente de nuestra economía. En: Ferrari, G. y Fernández López, M. (eds.). Raúl Prebisch Obras, 1919-1948. Tomo II. Fundación Raúl Prebisch, Buenos Aires, pp. 158-306.

Romer, C., 1992. What Ended the Great Depression. Journal of Economic History. 52 (4), 757-784.

Rosenberg, E. S., 1999. Financial missionaries to the world: The politics and culture of dollar diplomacy, 1900-1930. Duke University Press, Durham. Doi: $10.2307 / 2651684$.

Salera, V., 1941. Exchange control and the Argentine market. Columbia University, New York.

Sayre, R. A., 1948. Consumer's prices. 1914-1948. National Industrial Conference Board, Inc., New York.

Taylor, M., Taylor, A., 2004. The purchasing power parity debate. NBER Working Paper Series, Cambridge.

Temin, P., 2010. The Great Recession and The Great Depression. Daedalus. 139 (4), 115-124.

Temin, P., 1993. Transmission of the Great Depression. Journal of Economic Perspectives. 7 (2), 87-102.

The Economist, 2011. Lesons of the 1930s. There could be trouble ahead. The Economist, Briefing, 10-12-2011. Disponible en: https://econ.st/30Kfey3.

Thorp, R., 1998. Capítulo 4: Rupturas y experimentos: 1914-1945. En: Progreso, Pobreza y Exclusión. Una Historia Económica de América Latina En el siglo xx. Banco Interamericano de Desarrollo, Washington, pp. 103-133.

Thorp, R. (ed.). 1984. Latin America in the 1930s. Macmillan Press Ltd., London.

Williams, J. H., 1920. Argentine International Trade under Inconvertible Paper Money, 1880-1900. Harvard University Press, Cambridge. 